\title{
Childbirth information feeds for first time Malawian mothers who attended antenatal clinics
}

\section{Address Malata and Ellen Chirwa \\ Kamuzu College of Nursing, University of Malawi, P/Bag 1, Lilongwe. \\ Corresponding author, e-mail: addressmalata@kcn.unima.mw}

\begin{abstract}
Childbirth information received by mothers during antenatal period influences their satisfaction with the care during perinatal period. It is important for the midwives to know the type of information that satisfies their clients. This study found that the childbirth information that mothers received during antenatal period did not satisfy them. On the other hand, although some of the information received at home was useful, some information had potential to cause fear, anxiety and ill health on the mothers. The information needs of the clients were taken into account when designing a childbirth program for Malawi.
\end{abstract}

\section{Aim}

The specific objective of the study was to identify childbirth information needs of Malawian women as perceived by Malawian mothers and midwives in order to design a childbirth education program.

\begin{abstract}
Methods
An exploratory, descriptive qualitative design was used to determine Malawian women's childbirth information needs. A total of 150 first time mothers who attended antenatal clinics at selected central, district and mission hospitals were interviewed.Four focus group discussions were conducted with four different types of midwives to identify their perceptions of the childbirth information needs of Malawian women. These discussions were complimented by individual interviews with experienced midwives who held key positions in government and non governmental health organizations.
\end{abstract}

\section{Results}

The content of the childbirth education program for pregnant mothers should include; care during pregnancy, danger signs during pregnancy, labor and after birth, the labor process and the postnatal care for the mother and the baby. Regarding cultural beliefs and taboos of childbirth, it was recommended that they should not be presented as a stand alone topic but be incorporated in other topics such as self care. Cultural beliefs and taboos vary with regions and tribes hence rather than generalizing, midwives should address specific issues prevalent in their work areas. The training manual should be translated into the vernacular language in view of the high illiteracy rate in the country.

\section{Conclusion}

The education program should address self-care during pregnancy, nutrition during pregnancy, common discomforts of pregnancy, danger signs of pregnancy, sexually transmitted diseases and preparation for delivery. The program should also address the labor process, possible complications during labor and birth, caesarean birth and non pharmaceutical pain relief measures in labor. It was also evident that the program should deal with self-care during postnatal period, exclusive breast feeding, care of the newborn baby, danger signs of puerperium, care of the newborn baby and family planning.

\section{Introduction}

Knowledge of childbirth information needs is critical for the development of intervention strategies for pregnant women. In the developed countries, some studies ${ }^{1}$ described the needs of a woman together with those of their families because knowledge of the mother's needs forms a basis for planning a childbirth program. Childbirth experience of mothers facilitates physical, social and emotional change.
It is apparent that most mothers receive insufficient and inappropriate information about pregnancy and hence there is a need to identify women's needs to ensure the provision of appropriate and adequate information ${ }^{1}$.

Another perspective associated with ascertaining information needs is that of comparing what pregnant women want to know with what health professionals perceive must be taught. Significant differences have been reported ${ }^{2}$ between clients and their information providers. Hence, there have been major implications on how nurses assess women's needs for specific perinatal information ${ }^{2}$. Women reported greatest interest in topics such as foetal development, nutrition, vitamins, travel, bottle feeding, danger signs during pregnancy, when to go to the hospital, medicines in labor, how to know when labor starts, effects of stress on pregnancy, rest and activities, discomforts in pregnancy, anaesthesia, natural childbirth, birth defects, bleeding in pregnancy and breast feeding ${ }^{2}$.

Providers on the other hand, felt clients would be more interested in topics such as use of forceps, breast-feeding, family violence and when to go to the hospital ${ }^{2}$. Interestingly, primiparous women expressed interest in all topics, while the multiparousexpressed interest in selected topics. Understanding the relationship between pregnant women's and providers' perceptions could help prenatal health educators meet clients' needs better while teaching topics they know are necessary and desirable ${ }^{2}$.

\section{Background}

In the developed countries, antenatal classes have been in existence for a century. Childbearing experience is a challenging experience for parents particularly mothers. Antenatal classes are believed to assist parents and relieve them from the challenging experience. Examination of social processes and interactions that promoted women's experiential knowledge of birth showed that childbirth education courses assisted women to acquire biomedical knowledge about childbirth ${ }^{3}$. Furthermore, social processes influenced knowledge acquisition and encouraged social interaction and experience sharing.

In developing countries, organized antenatal classes are referred to as childbirth education programs ${ }^{4}$. In response to high maternal mortality rate of 249 deaths per 10,000 live births in Thailand in the year 2000, a childbirth education program was developed and evaluated ${ }^{5}$. The study was conducted in a regional hospital where there was a systematic and well-planned health education program in place for pregnant women. When a childbirth training program was developed and evaluated, there were significant changes in women's behavior in preparing for pregnancy and delivery including in the postpartum period. The women reported high level of satisfaction with the topic that dealt with preparation for delivery but moderate satisfaction with topics on preparation for pregnancy, nutrition and breastfeeding ${ }^{5}$.

\section{Rationale}

The perceptions of mothers and midwives regarding childbirth information content are important in the development of an effective childbirth education program for pregnant women. Parents, in particular mothers have preconceived expectations about what they want to know 
about childbirth. Most importantly, in addition to pain relief and body care is the assurance of a safe outcome for both baby and the mother. These perceptions may differ with those of the midwives and hence resulting to mothers' dissatisfaction with the antenatal clinic services. In Malawi, the current childbirth information content did not take into account the women's needs, hence the basis for this study.

\section{Objectives}

The objective of the study wasto identify childbirth information needs of Malawian women as perceived by Malawian mothers and midwives in order to design a training program for midwives that would lead to mothers' satisfaction with antenatal services.

\section{Methods}

\section{Design}

An exploratory descriptive qualitative design was used to determine Malawian women's childbirth information needs. Using a scale ranging from extremely dissatisfied to extremely satisfied, women were asked how satisfied they were with the labor and birth information they received using a structured questionnaire. In addition, four focus group discussions were conducted with four different groups of midwives to identify their perceptions of information needs of Malawian women. The participants included midwifery educators and clinicians from four settings within Malawi. Individual interviews were conducted with ten experienced midwives who held key positions in government and non governmental health organizations.

\section{Study Population and size}

The study was conducted at several sites ranging from a district hospital to primary tertiary teaching hospitals. A total of 150 first time mothers in Malawi were asked how satisfied they were with the labor and birth information they received. The question only applied to items on which the mothers stated that they had received information. As for the midwives, focus group interviews took place at one school of nursing, one district hospital and two central hospitals. These sites were chosen to elicit a broad range of perspectives of childbirth information needs of Malawian women6. The first focus group discussion was conducted at Kamuzu College of Nursing with 8 midwives who were lecturers in Midwifery at Bachelor's degree level. The second focus group interview took place at Queen Elizabeth Central Hospital (GogoChatinkha Maternity Wing) and involved 9 participants. The wing has an antenatal clinic and ward, labor ward, postnatal ward, family planning and under five clinics. Individual interviews were conducted with the midwives and involved a total number of 10 people

\section{Inclusion and exclusion criteria}

The women interviewed were those that were first time mothers and were attending antenatal clinic education in selected health facilities of the country. The Midwifery educators at University level and practicing midwives with at least 2 years experience from government district and central hospitalswere interviewed. In addition midwives that held key posts in government and non governmental organizations were also interviewed.

\section{Data Collection}

The 150 women were recruited from Queen Elizabeth Central hospital, Kamuzu Central hospital and Mulanje
Mission hospital. Focus group interviews with midwives were conducted at one school of nursing, one district hospital and two central hospitals. These sites were chosen to represent a broad range of perspectives of childbirth information needs of Malawian women and assist in increasing the validity of the study's findings. The first focus group was conducted at Kamuzu College of Nursing with midwives who teach midwifery at the college. The second focus group discussion took place at Queen Elizabeth Central Hospital (Gogo Chatinkha maternity wing). The third focus group interview was held at Mulanje district hospital and the last discussion was held at Lilongwe Central Hospital.

The focus group discussions were complemented by individual key informant interviews which were held in a variety of settings at the participant's work places. The informants were senior and more experienced midwives who had also worked in the clinical area although most of them are now involved in midwifery education and administration. The key informants included, the controller of Preventive Health Services, the Program Managers for Safe Motherhood Initiative in Lilongwe and Blantyre, the training officer at the Nurses and Midwives Council of Malawi, Senior Lecturer in Maternal and Child Health at Kamuzu College of Nursing, Senior Lecturer in Medical and Surgical Nursing at Kamuzu College of Nursing, a senior Matron at Gogo Chatinkha Maternity Wing of Queen Elizabeth Central Hospital, a Senior Matron at the Bwaila Hospital in Lilongwe and the Matron for Mulanje District Hospital.

A focus group guide with semi-structured questions was used in the focus group discussions. The questions focused on childbirth education content and effective strategies for dissemination of information. The individual interviews were guided by semi-structured, open ended questions on childbirth education content and strategies for information dissemination.

\section{Analysis}

The data from focus group discussions and individual interviews was managed in Q.S.R NUDI*ST (Nonnumerical Unstructured Data: Indexing, Searching, and Theorizing). Audiotapes were transcribed verbatim and each typed transcript was checked against the audiotape as soon as each interview had taken place. The written transcripts from each interview were later during the analysis read and key words and significant statements highlighted throughout the script. The identified themes that emerged from each interview were reviewed and similar themes that emerged were grouped together.

\section{Results}

\section{Demographic data of the mothers}

The age of the 150 first time mothers ranged between 13 and 30 years with a mean of 19 years. The majority $(76 \%)$ of the women was married and was living with their husbands. Most participants (74\%) achieved a lower primary education and were not working. Antenatal attendance was high with about $77 \%$ of the participants having had attended antenatal clinic 3 to 4 times during their pregnancy. The mothers had two sources of information; the hospital and home. The information received from the hospital was grouped into four themes;information before labor, information during birth, birth information and information after birth. 


\section{Information received at the hospital}

The mothers indicated that the before labor information they receive included; signs of onset of labor, preparation for labor and birth, need for rest and exercise and importance of hospital birth. However, the participants complained that the emphasis at the hospital was on signs of onset of labor and the other topics were poorly covered. Concerning the information acquired during birth, the mothers indicated that they received information regarding positions during labor, crying during labor, breathing exercises during labor, what could go wrong during labor, nutrition during labor, monitoring labor progress, assistance that can be given during labor and the process of labor. The participants indicated that this was information was adequate and met their needs. Regarding information for birth, the participants' received the following information, which was also adequate; bearing down during birth and how actual birth occurs. Finally, only one topic was covered on information for after birth and dealt with breast feeding.The participants indicated that they also needed information on care of mother and baby after birth. These two topics were included in the childbirth education program.

\section{Information received at home}

The mothers received cultural based information at home from traditional counsellors, family and friends and these mainly comprised what the woman should or should not do during pregnancy. After content analysis the information was clustered into three themes: actions which prolong labour, actions which influence poor outcome for baby and actions which enhance labour.

According to cultural beliefs, the actions which prolong labour were; standing or sitting at the door, walking in the forward direction all the time and a sneak look through the window. These actions were considered to have a negative effect on the progress of labour. Women were encouraged to avoid anything that would delay the progress of labour. On the other hand, the actions which enhance labour were: taking traditional medicines which are believed to facilitate onset of labour. Women take the drug at home before they get to the hospital to facilitate labour but are advised not to reveal to hospital staff for fear of being chastised. Under the third theme of actions that cause poor outcome for the baby, the women were taught that if a pregnant woman swallows saliva when she sees a lame person, she would give birth to a baby with some form of disability. Therefore, the women were discouraged from these actions to prevent poor outcomesfor the baby.

In addition the women indicated that they needed extra information which was neither given at the hospital nor at home. The needed information was grouped into three themes; information for the mother, information regarding labor and birth, and, information for the new born baby. Regarding information for the mother, the women were interested to know their rights during labor and birth, and, the process of labor and birth. The women were also interested to know about what could go wrong during labor and birth, indications for interventions with focus on caesarean birth, the process of labor, and pain relieving measures. Being first time mothers they also wanted to know how the newborn baby looks like and what the baby is capable of doing.These topics were important so that women were prepared for the labor and birth experience. These areas were hence included in the childbirth education program.

\section{Discussion}

The ages of the first time mothers in this study show that most girls are becoming first time mothers at a young age. In Malawi, it is reported that teenage pregnancies are one of the major health challenges signifying that marriage and child bearing are starting at an early teen age ${ }^{7}$. The results therefore imply that childbirth Education Program should also incorporate issues of adolescence through tailor made training programs. Studies in the developed countries are consistent with the results of this study as they also show that teenage mothers are at great risk for pregnancy, labor and postpartum complications $s^{8,9}$. Therefore, there is a need to provide age appropriate information to adolescent mothers. It is crucial that the information needs of adolescents on physical, psychological and sociological needs of pregnancy, labor, birth and postpartum period be met through childbirth education program.

The education level of the women was a major consideration in the development and implementation of the childbirth education program. For the midwives, the program was developed in English but had to be translated into the vernacular language for the mothers. Education level of participants is important as it influences the ability to seek, understand, and appreciate the information received? Furthermore, the education level of the mother influences their attendance of antenatal classes and acceptance of advice on the need for hospital care contrary to the cultural beliefs.

\section{Perceived childbirth information needs}

During pregnancy, mothers develop expectations concerning their anticipated labor and birth. These expectations play an important role in determining mothers' responses to the childbirth experience ${ }^{10,11,12}$. The results in this study are supported by other studies that findings that most prenatal education programs claim to be based on learning needs of pregnant women but in reality the learning needs are based on the perceptions of health professionals ${ }^{13,14}$.

Other studies conducted in developed countries support the findings of this study that the content of childbirth education program include: anatomy and physiology of the reproductive system, normal labor and delivery process, caesarean birth, labor and birth drugs and their effects, relaxation and breathing techniques, normal progress of labor and role of the woman in labor ${ }^{15}$ pain and pain relief ${ }^{16}$ coping with the stress of labor ${ }^{17,18}$ labor and delivery, breathing and relaxation, maternity tour, hospital procedures and legal information ${ }^{19}$ and stages of labor ${ }^{20}$.

\section{Ideas presented by women for improving current methods of providing information}

Malawian women identified several issues which focused on the practical methods of information delivery. One of the recommendations was that the childbirth education program should be developed with specific information targeting both an individual and groups of mothers. This recommendation is in agreement with the findings reported in a number of studies ${ }^{4,21,22}$. Furthermore, it was recommended that the midwives be oriented in the new training program. Emphasis was made to advise the midwives to create an environment for interactive learning through question and answer sessions. Finally, it was recommended that the midwives should be 
friendly and use the language that is appropriate for the mothers, as such avoid technical or medical terminology ${ }^{23}$.

\section{Information received by women}

Overall the results of this study show that the majority of the mothers in this study had received information concerning labor and birth. However, the information regarding other equally important areas was either too little or not available. Of concern was the finding that some of the women were not given any specific information about labor and birth. Other studies from developed countries have also identified similar information needs ${ }^{24,25}$. The major reason for this was that the current childbirth training program mainly focused on HIV and AIDS as well as preparation for birth, which included clothes and candles for lighting in case lights went off. This shows that only one aspect of labor and birth information received a high degree of emphasis from the midwives. The rest of the labor and birth information received little or no attention at all.

Some of the information given to the mothers at home, though based on socio-cultural beliefs was helpful. For example, during labor the mother was encouraged to walk around, if she could. This advice makes medical sense as ambulation encourages the progress of labor ${ }^{26,27}$. The cultural rationale for walking about during labor was to prevent the baby from delaying its birth because the exercises stimulate the unborn babies to be active during the birth process. On the other hand, some of the information given to mothers at home had potential to cause maternal anxiety. For example, the advice against sitting or standing at a door so that the baby would not stop at birth pathway during birth. Field notes indicated that some midwives encouraged beliefs in traditional information. One mother told a story of a midwife at an antenatal visit who told her to move away from a door where she was standing. The midwife even asked the mother whether or not she had received this information at home. The everyday life of a woman involves walking through different types of doors, complying with this belief could be very difficult for the mother. If the mother experienced prolonged labor, she could easily believe that her experience was attributed to the beliefs.

Furthermore, some of the information given at home such as the use of traditional medicine may have been harmful to some mothers. The strength and composition of the drug is not known, and unless a scientific study is undertaken on the drug, it is difficult to advocate or discourage use of the traditional drugs. Anecdotal evidence from midwives indicated that some mothers who took the drug had precipitate labor and/or ruptured uterus.

\section{Conclusion}

There was a need to develop a childbirth education program in a participatory manner by incorporating the needs of mothers especially those that were falling pregnant for the first time. The cultural information given to pregnant mothers contained unsafe practices that needed to be discouraged and some useful information that needed to be encouraged. The overall training program should comprise three sections. Section one should address antenatal care, selfcare during pregnancy, nutrition during pregnancy, common discomforts of pregnancy, danger signs of pregnancy, sexually transmitted diseases and preparation for delivery. The second section should address the labor process, possible complications during labor and birth, caesarean birth and non pharmaceutical pain relief measures in labor. The last section should deal with self-care during postnatal period, exclusive breast feeding, care of the newborn baby, danger signs of puerperium, care of the newborn baby and family planning. Midwives should be trained on the new program and there is a need for a monitoring process to see if the new childbirth education program is influencing maternal seeking behavior of pregnant women in Malawi.

\section{Conflict of Interest}

None of the authors had any known conflict of interest in the publication of this paper.

\section{Ethical consideration}

Ethical approval to conduct this study was obtained from the Committees for Conduct of Ethical Research at Edith Cowan University in Australia and the College of Medicine's Research and Ethics Committee (COMREC) in Malawi.

\section{Acknowledgements}

This study was funded by Edith Cowan University through an International Postgraduate Research Scholarship that was granted to the first author as part of PhD studies in Nursing. Sincere gratitude is due to the staff of Kamuzu College of Nursing for their support. This paper was edited by Dr. Alfred Maluwa, the Research Director at Kamuzu College of Nursing.

\section{References}

1. Blackford, K. A., Richardson, H., \& Grieve, S. Prenatal education for mothers with disabilities. Journal of Advanced Nursing, 2000, 32(4): 898-904.

2. Freda, M., Anderson, F., Damus, K., \& Merkatz, I. What pregnant women want to know: A comparison of client and providers' perceptions. Journal of Obstetric, Gynecological and Neonatal Nursing, 1993,22(8): 237-242.

3. Ketler, S. K. Preparing for motherhood: Authoritative knowledge and the undercurrents of shared experience in two childbirth education courses in Cagliari, Italy. Medical Anthropology Quarterly, 2000, 14(2): 138-158.

4. Williams, M., \& Booth, D. Antenatal education: Guidelines for teachers1980, (2nd ed.). Edinburgh: Longman Group Limited.

5. Thassri, J., Kala, N., Chusington, L., Phongthananasarn, J., Boonsrirat, S., \& Jirojwong, S. The development and evaluation of a health education programme for pregnant women in a regional hospital, southern Thailand. Journal of Advanced Nursing, 2000, 32(6), 14501458 .

6. Morse, J. Qualitative nursing research: 1991. Newbury Park: Sage Publication.

7. National Statistical Office, Zomba, Malawi. Malawi National Population Policy, 1992.

8. Breedlove, G. K. A description of social support and hope in pregnant and parenting teens receiving care from a doula.2001. Unpublished $\mathrm{PhD}$, University of Missouri, Kansas City.

9. Flemming, S. Adolescent pregnancy in Australia: Perspectives and problems. Modern Medicine of Australia, 1990, 5: 64-72.

10. Beger, D., \& Beaman, M. L. Childbirth education curriculum: An analysis of parent and educator choices. Journal of Perinatal Education, 1996, 5 (4): 29-35.

11. Evans, S., \& Jeffrey, J. Maternal learning needs during labour and delivery. Journal of Obstetric, Gynecologic, \& Neonatal Nursing,1995, 24(3): $235-240$.

12. Spitzer, R. Meeting consumer expectations. Nursing Administration, 
1988:12(3), 31-39.

13. Sullivan, P. L. Felt learning needs of pregnant women. Canadian Nurse, 1993, 89(1), 42-45.

14. Wolley, D., \& Roberts, J. Teaching about second stage: Implications for practice. Journal of Perinatal Education, 1995: 4(4), 45-48.

15. Hallgren, A., Kihlgren, M., Norberg, A., \& Forslin, L. Women's perceptions of childbirth and childbirth education before and after education and birth. Midwifery, 1995, 11: 130-137.

16. Green, J. Expectations and experiences of pain in labour: Findings from a large perspective study. Birth, 1993, 20(2): 65-72.

17. Maloney, R. Childbirth education classes. Journal of Obstetric, Gynecologic, \& Neonatal Nursing, 1985, 4: 245-248.

18. Simkin, P. Just another day in a woman's life? Part 1. Women's long term perceptions of their first childbirth experience. Birth, 1991, 18(4): 203-211.

19. Mollart, L. Pregnant teenagers antenatal education research.1995, Paper presented at the Australian College of Midwives Inc. 9th Biennial Conference, Sydney.

20. Jacoby, A. Mothers' views about information and advice on pregnancy and childbirth: Findings from a national study. Midwifery, 1988, 4: 103-110.
21. Redman, B. K. The process of patient education1993,(7th ed.). St. Louis: Mosby Year Book.

22. Schneider, Z. Antenatal education classes in Victoria: What women said. Australian Journal of Midwifery, 2001,14(3): 14-21.

23. Renkert, S., \& Nutbeam, D. Opportunities to improve maternal healthy literacy through antenatal education: an exploratory study. Health Promotion International, 2001, 16(4): 381-388.

24. Callaghan, L., Jones, J., \& Leonard, L. Evaluation of a childbirth and parenting education service. Birth Issues, 2001, 10(2): 41-49.

25.Corwin, A. Integrating preparation for parenting into childbirth education. Journal of Perinatal Education, 1995,8(1): 22-28.

26. Enkin, M., Keirse, M., Renfrew, M., \& Neilson, J. A Guide to Effective Care in Pregnancy \& Childbirth (2nd ed.). 1995. Newyork: Oxford University Press Inc.

27. Beischer, N. A., Mackay, E. V., \& Purcal, N. K. Care of the pregnant woman and her baby1989, (2nd ed.). Sydney: Saunders/Brailliere Trindall. 